\title{
Sequential cleavage of insulin receptor by calpain 2 and $\gamma$-secretase impairs insulin signalling
}

\author{
Tomoyuki Yuasa ${ }^{1,2}$ - Kikuko Amo-Shiinoki ${ }^{1,3}$ - Shuhei Ishikura ${ }^{1,4}$. \\ Mitsuyoshi Takahara $^{5,6}$ - Takaaki Matsuoka ${ }^{5}$. Hideaki Kaneto ${ }^{5,7}$. \\ Akio Kuroda $^{8,9} \cdot$ Munehide Matsuhisa $^{8,9} \cdot$ Seiichi Hashida ${ }^{10}$
}

Received: 8 July 2016 / Accepted: 8 August 2016/Published online: 30 September 2016

(C) Springer-Verlag Berlin Heidelberg 2016

\begin{abstract}
Aims/hypothesis Soluble insulin receptor (sIR), the ectodomain of the insulin receptor (IR), has been detected in human plasma and its concentration paralleled that of blood glucose. We have previously developed an in vitro model using HepG2 liver-derived cells, which mimics changes in sIR levels in plasma from diabetic patients and shows that calcium-dependent proteases cleave IR extracellularly (a process known as shedding). The present study aimed to reveal the mechanisms of IR cleavage.

Methods Using the in vitro model, we investigated the molecular mechanisms of IR cleavage, which is accelerated by highglucose treatment. We also analysed the relationship between
\end{abstract}

IR cleavage and cellular insulin resistance, and the correlation between plasma sIR levels and insulin sensitivity, which was assessed by the euglycaemic-hyperinsulinaemic clamp technique.

Results Here, we determined that calpain 2, which is secreted into the extracellular space associated with exosomes, directly cleaved the ectodomain of the $\operatorname{IR} \beta$ subunit $(\operatorname{IR} \beta)$, which in turn promoted intramembrane cleavage of IR $\beta$ by $\gamma$-secretase. IR cleavage impaired insulin signalling and the inhibition of IR cleavage (by knockdown of calpain 2 and $\gamma$-secretase), restored IR substrate-1 and Akt, independent of IR. Furthermore, the glucose-lowering drug, metformin, prevented IR cleavage accompanied by inhibition of calpain

Kikuko Amo-Shiinoki and Shuhei Ishikura contributed equally to this study.

Electronic supplementary material The online version of this article (doi:10.1007/s00125-016-4102-5) contains peer-reviewed but unedited supplementary material, which is available to authorised users.

Tomoyuki Yuasa

yuasa.tomoyuki@tokushima-u.ac.jp

1 Division of Molecular Genetics, Institute for Enzyme Research, Tokushima University, Tokushima, Japan

2 Present address: Department of Community Medicine for Diabetes and Metabolic Disorders, Tokushima University Graduate School, 3-18-15 Kuramoto-cho, Tokushima-shi, Tokushima 770-8503, Japan

3 Present address: Department of Diabetes Research, Yamaguchi University School of Medicine, Yamaguchi, Japan

4 Present address: Department of Cell Biology, Faculty of Medicine, Fukuoka University, Fukuoka, Japan
5 Department of Metabolic Medicine, Osaka University Graduate School of Medicine, Osaka, Japan

6 Present address: Department of Diabetes Care Medicine, Osaka University Graduate School of Medicine, Osaka, Japan

7 Present address: Department of Diabetes, Endocrinology and Metabolism, Kawasaki Medical School, Okayama, Japan

8 Diabetes Clinical Research and Development Center, Tokushima University, Tokushima, Japan

9 Present address: Diabetes Therapeutics and Research Center, Institute for Advanced Medical Sciences, Tokushima University, Tokushima, Japan

10 Human Life Science, Tokushima Bunri University, Tokushima, Japan 
2 release in exosomes, and re-established insulin signalling. In patients with type 2 diabetes, plasma sIR levels inversely correlated with insulin sensitivity.

Conclusions/interpretation Sequential cleavage of IR by calpain 2 and $\gamma$-secretase may contribute to insulin signalling in cells and its inhibition may be partly responsible for the glucose-lowering effects of metformin. Thus, IR cleavage may offer a new mechanism for the aetiology of insulin resistance.

Keywords Calpain 2 - Exosome $\cdot$ Insulin resistance · Metformin $\cdot$ Shedding $\cdot$ Soluble insulin receptor $\cdot$ Type 2 diabetes

\begin{tabular}{|c|c|}
\hline \multicolumn{2}{|c|}{ Abbreviations } \\
\hline AMPK & 5' AMP-activated protein kinase \\
\hline BAPTA-AM & $\begin{array}{l}O, O^{\prime} \text {-Bis [2-aminophenyl] } \\
\text { ethyleneglycol- } N, N, N^{\prime}, N^{\prime} \text {-tetraacetic acid } \\
\text { tetraacetoxymethyl ester }\end{array}$ \\
\hline DON & Deoxynorleucine \\
\hline ERK & Extracellular signal-regulated kinase \\
\hline $\operatorname{ICD}-\mathrm{IR} \beta$ & $\begin{array}{l}\text { Intracellular C-terminal domain of the } \\
\beta \text { subunit of the insulin receptor }\end{array}$ \\
\hline IR & Insulin receptor \\
\hline $\operatorname{IR} \beta$ & IR $\beta$ subunit \\
\hline$O-G l c N A c$ & O-linked $\mathrm{N}$-acetylglucosamine \\
\hline sIR & Soluble insulin receptor \\
\hline siRNA & Small interfering RNA \\
\hline TSAP6 & Tumour suppressor-activated pathway 6 \\
\hline
\end{tabular}

\section{Introduction}

Insulin signalling exerts pleiotropic effects on cellular metabolism, growth and differentiation. Impaired insulin signalling causes insulin resistance $[1,2]$ and several mechanisms have been postulated to explain its pathogenesis [3]. Nevertheless, the causative molecule of human insulin resistance has not been identified [4].

Insulin binds to the insulin receptor (IR) on the plasma membrane and activates tyrosine kinase, which consequently initiates the intracellular signalling pathway [5]. We found that soluble IR (SIR), the ectodomain of IR, exists in human plasma and its concentration paralleled that of blood glucose [6]. We also found that human primary hepatocytes and endothelial cells generated sIR and from this we developed an in vitro model that mimicked changes in sIR levels in plasma from patients with diabetes [7]. This model demonstrated that IR underwent proteolytic cleavage extracellularly, on the plasma membrane. However, it remains to be determined how IR is cleaved.

The soluble ectodomains of several membrane receptors have been detected in plasma samples. Most of these ectodomains are released by proteolytic cleavage of the membrane receptor, a process also known as shedding. The physiological implications of receptor cleavage are diverse. In some cases, the soluble receptor may function as a decoy receptor, which inhibits the activities mediated by binding of their ligands $[8,9]$. Membrane receptor cleavage can also downregulate ligand signalling by decreasing the amount of intact receptor localised on the cell membrane [10]. In other cases, ectodomain shedding is followed by intramembrane proteolysis and translocation of the intracellular domain to the nucleus, where it acts as a transcription factor [11, 12]. However, the physiological role underlying IR cleavage has not been defined.

Here, we present the molecular mechanisms of sIR generation and the biological role of IR cleavage in impaired insulin receptor signalling in cells cultured in high-glucose conditions. We also analysed the functional role of biguanides in IR cleavage and investigated the correlation between plasma sIR levels and insulin sensitivity in patients with type 2 diabetes.

\section{Methods}

Materials The following antibodies and reagents were purchased from Cell Signaling Technology (Beverly, MA, USA): (1) rabbit monoclonal antibodies against presenilin 1 (D39D1), His-tag (D3I10), p-IRS-1 (Ser612; C15H5), p-Akt (Ser473; D9E), p-Akt (Thr308; C31E5E), Akt (C67E7), p-p44/42 mitogen-activated protein kinase (MAPK) (extracellular signal-regulated kinase [ERK] 1/2; Thr202/Tyr204; D13.14.4E) and p44/42 MAPK (137 F5); (2) rabbit polyclonal antibodies against calpain 2 large subunit (M-type) and p-IRS1 (Ser636/639); and (3) mouse monoclonal antibodies against the IR $\beta$ subunit (IR $\beta$; L55B10) and p-tyrosine (P-Tyr-100). Rabbit polyclonal antibodies against CD63 were purchased from Abcam (Cambridge, UK). Rabbit polyclonal antibodies against IRS1 (06-248), recombinant rat calpain-2 and MG132 were purchased from Merck-Millipore (Billerica, MA, USA). Rabbit polyclonal antibodies against IR $\beta$ (C-19 and SC-711) were purchased from Santa Cruz Biotechnology (Santa Cruz, CA, USA). Mouse monoclonal antibodies against $\beta$-tubulin (TUB 2.1) and deoxynorleucine (DON), L-685,458 and metformin (1,1-dimethylbiguanide hydrochloride) were purchased from Sigma-Aldrich ( $\mathrm{St}$ Louis, MO, USA). $O, O^{\prime}$-Bis [2-aminophenyl] ethyleneglycol- $N, N, N^{\prime}, N^{\prime}$-tetraacetic acid, tetraacetoxymethyl ester (BAPTA-AM) were purchased from Dojindo (Kumamoto, Japan). Buformin hydrochloride was purchased from Wako (Osaka, Japan). Recombinant human EGF was purchased from Pepro Tech (Rocky Hill, NJ, USA). Mouse monoclonal antibodies against the IR $\alpha$ subunit (5D9) were validated [13] and donated by R. A. Roth (Stanford School of Medicine, Stanford, CA, USA). All other antibodies were 
validated by their manufacturers. All other reagents used were of analytical grade.

Cell culture HepG2 cells (American Type Culture Collection, Manassas, VA, USA) were grown as previously described [7]. In brief, HepG2 cells were grown in DMEM basal glucose (Life Technologies, Gaithersburg, MD, USA) supplemented with $10 \% \mathrm{FBS}$ (vol./vol.) and were cultured at $37^{\circ} \mathrm{C}$ in $5 \%$ $\mathrm{CO}_{2}$. MG132 was used to prevent proteasome degradation of the intracellular C-terminal domain of IR $\beta$ (ICD-IR $\beta$ ). Mycoplasma contamination was not determined.

\section{Immunoprecipitations and western blot analysis Cell} lysates were prepared and equal amounts of protein were subjected to immunoprecipitation with specific antibodies as previously described [14]. In brief, cell lysates were prepared with lysis buffer (1\% (wt/vol.) Nonidet P-40, $50 \mathrm{mmol} / \mathrm{l}$ Tris-HCl, pH 7.4, $140 \mathrm{mmol} / \mathrm{l} \mathrm{NaCl}, 10 \mathrm{mmol} / \mathrm{l}$ EDTA, $20 \mathrm{mmol} / \mathrm{l}$ sodium fluoride, $1 \mathrm{mmol} / \mathrm{l}$ sodium orthovanadate, $20 \mathrm{mmol} / \mathrm{l} \beta$-glycerophosphate, $1 \mathrm{mmol} / \mathrm{l}$ phenylmethylsulfonyl fluoride, $4 \mu \mathrm{g} / \mathrm{ml}$ aprotinin, $4 \mu \mathrm{g} / \mathrm{ml}$ leupeptin and $4 \mu \mathrm{g} / \mathrm{ml}$ Pepstatin A), and the precipitate was removed by centrifugation at $16,100 \times g$ for $20 \mathrm{~min}$ at $4^{\circ} \mathrm{C}$. Equal amounts of protein were subjected to immunoprecipitation with specific antibodies and Protein A-Sepharose CL-4B or Protein G-Sepharose 4 Fast Flow (GE Healthcare, Little Chalfont, UK). Extracts from the immunoprecipitates or equal amounts of cell lysates were resolved by SDS-PAGE, transferred onto a nitrocellulose membrane and probed with specific antibodies. All primary antibodies were used at a 1:1000 dilution. Proteins were visualised using secondary antibody (horse-radish peroxidase conjugated goat anti-rabbit or mouse IgG, at 1:2000 dilution: Medical \& Biological Laboratories, Nagoya, Japan) and enhanced chemiluminescence (ECL Prime; GE Healthcare). A representative immunoblot for each western blot analysis is shown.

Ultrasensitive ELISA for human sIR We used an ultrasensitive ELISA (immune complex transfer enzyme immunoassay) to measure the levels of human sIR, as previously described [15]. In brief, the sIR-antibody immune complexes were transferred from one solid phase to another solid phase. Two kinds of monoclonal antibodies against the human specific IR $\alpha$ subunit were used: 5D9 (gifted from Medical \& Biological Laboratories) and 83-7 (purchased from Lab Vision, Fremont, CA, USA). The amount of intact IR in cell lysates was also determined using this assay. The net increase in IR cleavage was calculated by dividing the amount of sIR in the medium with that of IR in cell lysates, relative to that in control cells.

siRNA knockdown Ambion small interfering (si)RNAs for human CAPN2 (-1, S319; -2, S320; -3, S321) and PSEN1 (-1, S112; -2, S113) and the negative control (no. 1) siRNA were purchased from Thermo Scientific (Waltham, MA, USA). The knockdown protocol using these siRNAs has been previously described [7]. In brief, HepG2 cells were transfected with the indicated siRNAs $(100 \mathrm{nmol} / \mathrm{l})$. For insulin stimulation, the medium was replaced every $24 \mathrm{~h}$ with DMEM containing 5.5 or $16.5 \mathrm{mmol} / \mathrm{l}$ glucose plus $1 \%$ FBS (vol./vol.), which was followed by incubation in serum-free DMEM for $1 \mathrm{~h}$.

Isolation of exosomes from HepG2 culture medium Exosomes from the HepG2 culture medium were isolated using total exosome isolation Reagent (from cell culture media) (Thermo Scientific), according to the manufacturer's instructions with minor modifications. In brief, HepG2 cells were seeded in $10 \mathrm{~cm}$ dishes and incubated with DMEM containing 5.5 or $16.5 \mathrm{mmol} / \mathrm{l}$ glucose and 1\% FBS (vol./vol.) with or without $3 \mathrm{mmol} / \mathrm{l}$ metformin or $5 \mu \mathrm{mol} / \mathrm{l} \mathrm{DON}$ for $72 \mathrm{~h}$. After washing the cells with DMEM, $10 \mathrm{ml}$ DMEM without serum was added to each dish for an additional $24 \mathrm{~h}$. The cell media were centrifuged at $10,000 \mathrm{~g}$ for $30 \mathrm{~min}$ to remove cells and debris.

Calpain activity assay To measure the proteolytic activity of calpain 2, recombinant IR containing His-tag at the C-terminal of the IR $\beta$ ectodomain (Sino Biological, Beijing, People's Republic of China) was used as a substrate. Recombinant calpain $2(2 \mu \mathrm{g})$ (Merck-Millipore) was incubated with the substrate $(1.5 \mu \mathrm{g})$ in $20 \mathrm{mmol} / \mathrm{l}$ Tris- $\mathrm{HCl}(\mathrm{pH} 7.4), 1 \mathrm{mmol} / \mathrm{l}$ EGTA, with or without $3 \mathrm{mmol} / 1 \mathrm{CaCl}_{2}$, at $25^{\circ} \mathrm{C}$ for $20 \mathrm{~min}$. Reactions were stopped by adding SDS sample buffer. Proteolytic activity was evaluated by western blotting using antibodies against His-tag, in which disappearance of the Histagged IR $\beta$ ectodomain indicated its cleavage by calpain 2 . Coomassie staining was performed according to a standard protocol [16].

Euglycaemic-hyperinsulinaemic clamp study Sixteen Japanese participants with type 2 diabetes were recruited. The patients with advanced diabetic complications, such as unstable retinopathy and severe kidney dysfunction, and severe liver dysfunction were excluded. Their characteristics are summarised in the electronic supplementary material (ESM) Table 1. A euglycaemic-hyperinsulinaemic clamp study was performed in each patient after $>1$ week of intensive insulin therapy to achieve target blood glucose levels (pre-meal blood glucose $<7 \mathrm{mmol} / 1$ and $2 \mathrm{~h}$ post-meal blood glucose $<10 \mathrm{mmol} / \mathrm{l})$. The target plasma glucose and insulin levels during the euglycaemic-hyperinsulinaemic clamps were $5.5 \mathrm{mmol} / \mathrm{l}$ and $600 \mathrm{pmol} / \mathrm{l}$, respectively. When the exogenous glucose infusion rate reached a steady state, we determined insulin sensitivity as the metabolic clearance rate of glucose, which was calculated as the average endogenous glucose infusion rate divided by glucose concentration. We also measured plasma sIR concentrations at the start of the glucose clamp test to assess insulin sensitivity simultaneously. 
Pearson's correlation coefficient was used to determine the association between sIR level and metabolic clearance rate of glucose. The study was approved by the Ethical Committee for Human Studies at Tokushima University Hospital. Written informed consent was obtained from each patient after receiving a detailed written explanation of the study.

Statistical analysis No data were excluded and all results presented were substantiated by repeated experiments. Experiments were performed and analysed in a blinded fashion when possible. Values are presented as mean \pm SEM. Data involving more than two groups were analysed with ANOVA significance levels were set at $<0.05,<0.01$ and $<0.001$.

\section{Results}

Calpain 2 cleaves the extracellular domain of IR We have previously reported that the putative membrane protease that is assigned to cleave IR was dependent on calcium ions [7]. As a calcium-dependent protease, calpain performs proteolytic processing rather than degradation, and participates in a variety of biological events. To test the possibility that $\operatorname{IR} \beta$ is a calpain substrate, we analysed it using the calpain database (Calpain for Modulatory Proteolysis Database [CaMPDB]) [17]. The result showed that IR $\beta$ has some predicted sequences for calpain proteolysis, which are located on the $\mathrm{N}$-terminal side of the transmembrane domain in the $\beta$ subunit. Among the human calpains, calpain 2 is expressed extracellularly and intracellularly, making it a candidate for IR cleavage. Knockdown of calpain 2 using siRNA significantly decreased the amount of sIR in the medium (Fig. 1a) and inhibited the high-glucose-induced proteolytic effect on IR cleavage (Fig. 1b). In vitro, the calpain activity assay demonstrated that the $\mathrm{C}$-terminal His-tag of the recombinant IR $\beta$ ectodomain was removed in a calcium ion-dependent manner (Fig. 1c). Moreover, the IR $\beta$ ectodomain in which the His-tag was deleted could be observed in the Coomassie-stained gel (Fig. 1c). These results demonstrate that calpain 2 directly catalyses IR cleavage at a specific site of the IR $\beta$ ectodomain.

Sequential cleavage of IR by calpain 2 and $\gamma$-secretase A previous report has demonstrated that regulated intramembrane proteolysis by $\gamma$-secretase is preceded and regulated by an initial distinct cleavage in a process termed ectodomain shedding [18]. Knockdown of calpain 2 decreased the accumulation of ICD-IR $\beta$ (Fig. 1d), which has been shown to be digested by $\gamma$-secretase [7]. The high-glucose-induced accumulation of ICD-IR $\beta$ was also decreased by EGTA, but not by the intracellular calcium chelator, BAPTA-AM (Fig. 1e). Collectively, IR proteolysis by calpain 2 occurs in the extracellular space and is sequentially followed by intramembrane cleavage by $\gamma$-secretase (schematic shown in Fig. 1f).
Exosome-associated calpain $\mathbf{2}$ is released into cell media Our findings demonstrate that IR cleavage performed by calpain 2 occurs extracellularly. Because exosomes have been found to be released into HepG2 cell medium [19], we investigated whether calpain 2 is secreted into the extracellular space associated with exosomes. We found that high-glucose treatment increased the amount of calpain 2 in the HepG2 culture medium; however, there was no significant difference in the amount of exosomes between basal and high-glucose treatment (as indicated by CD63 levels in Fig. 2a).

O-linked $\mathrm{N}$-acetylglucosamine $(\mathrm{O}$-Glc $N A \mathrm{c})$ modification has been shown to be involved in the glucose dependency of IR cleavage [7]. Chemical inhibition of $O$-GlcNAc modification by DON suppressed the increment of calpain 2 relative to the reduction in exosomes, suggesting that calpain 2 release is modified by $O$-GlcNAcylation (Fig. 2b). Additionally, highglucose treatment significantly decreased intracellular calpain 2 expression, which was recovered by DON treatment (Fig. 2b). These results indicated that $O$-GlcNAc modification facilitated exosome-related calpain 2 release, accompanied by a decrease in intracellular calpain 2 expression, and that its inhibition restored the amount of calpain 2 in the cells.

IR cleavage impairs insulin receptor signalling To elucidate how cellular physiology was impacted by IR cleavage, the relationship between IR cleavage and insulin signalling in cells was analysed. Glucose at $\geq 11 \mathrm{mmol} / \mathrm{l}$ significantly induced IR cleavage (Fig. 3a), and at higher concentrations $(\geq 16.5 \mathrm{mmol} / \mathrm{l}$ ) glucose decreased the phosphorylation levels of Akt and ERK, two representative components of the insulin signalling pathway (Fig. 3b).

A previous study has reported that high glucose suppresses insulin signalling in HepG2 cells [20]. It has been subsequently reported that Akt1 and Akt2 are modified by $O$-GlcNAc, which attenuates Akt phosphorylation [21]. Akt is a component of various growth factor signalling pathways. Phosphorylation of Akt by EGF was not inhibited by a high glucose concentration (Fig. 3c), indicating that, in our model, direct $O$-GlcNAc modification is not responsible for the decreased phosphorylation of Akt, and that IR cleavage may cause selective dampening of insulin signalling.

EGTA extracellularly inhibits the calcium-dependent protease that cleaves IR [7]. EGTA partially rescued the high-glucose-induced suppression of insulin-induced Akt phosphorylation (Fig. 3d). Furthermore, knockdown of calpain 2 restored Akt phosphorylation (Fig. 3e). Moreover, knockdown of presenilin 1, which contains the active site of $\gamma$-secretase [22], enhanced Akt phosphorylation (Fig. 3f). These results demonstrate that sequential cleavage of IR is involved in the impaired insulin signalling induced by high glucose in cells. 


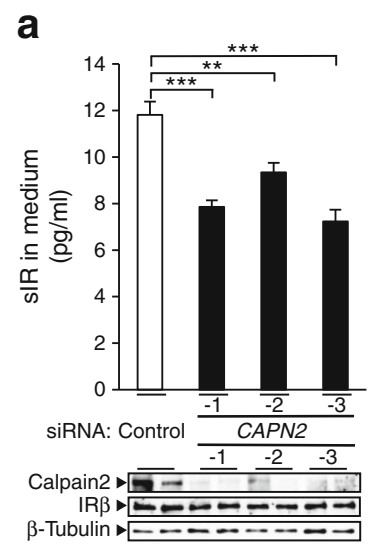

b $\begin{aligned} \text { Glc }(\mathrm{mmol} / \mathrm{l}): & \frac{5.5}{16.5} \\ \text { siRNA: } & \text { Control } \frac{\text { CAPN2 }}{-2}-3 \\ \text { Calpain } 2 & \end{aligned}$

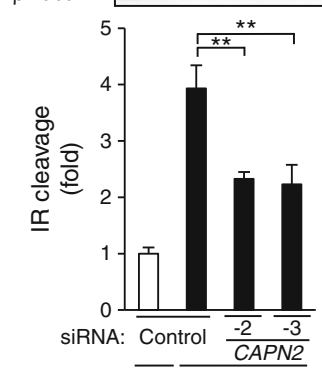

C

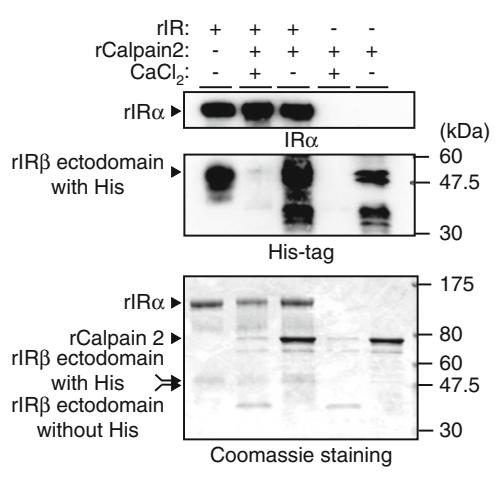

\section{d}

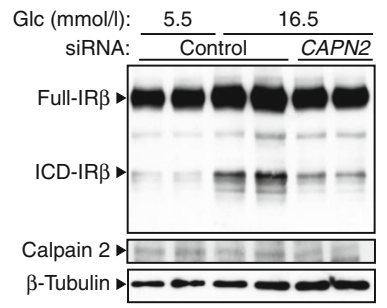

e

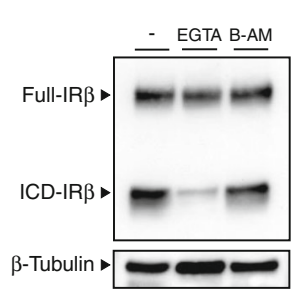

f

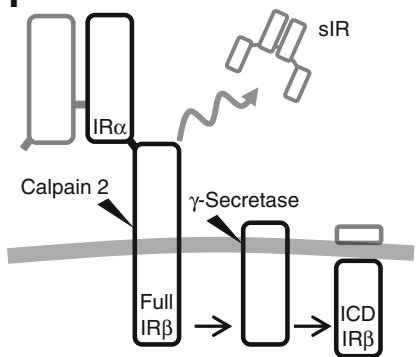

Fig. 1 Sequential cleavage of IR by calpain 2 and $\gamma$-secretase. (a) Role of calpain 2 on sIR generation. Following knockdown of calpain 2, cells were treated with $5.5 \mathrm{mmol} / \mathrm{l}$ glucose for $48 \mathrm{~h}$. Subsequently, cells were incubated for an additional $24 \mathrm{~h}$ and media and cell lysates were prepared $(n=6)$. (b) IR cleavage relative to control $(5.5 \mathrm{mmol} / 1$ glucose). Following knockdown of calpain 2, cells were treated with 5.5 or $16.5 \mathrm{mmol} / \mathrm{l}$ glucose for $48 \mathrm{~h}$. Subsequently, cells were incubated for an additional $24 \mathrm{~h}$ and media and cell lysates were prepared $(n=6)$. (c) In vitro calpain activity, as demonstrated by immunoblotting of IR $\alpha$ and His-tag $(n=3)$. (d) Sequential cleavage of IR. Following knockdown of calpain 2 and incubation with glucose for $48 \mathrm{~h}$, cells were incubated in serum-free DMEM containing $10 \mu \mathrm{mol} / 1 \mathrm{MG} 132$ for an additional $24 \mathrm{~h}$ $(n=3)$. (e) Extracellular cleavage of IR. Following incubation with $16.5 \mathrm{mmol} / 1$ glucose for $72 \mathrm{~h}$, cells were incubated in serum-free DMEM containing $5 \mu \mathrm{mol} / 1 \mathrm{MG1} 2$ with $5 \mathrm{mmol} / \mathrm{l}$ EGTA or $5 \mu \mathrm{mol} / \mathrm{l}$ BAPTA-AM for an additional $24 \mathrm{~h}(n=5)$. (f) Schematic representation of the proposed sequential cleavage model of IR by calpain 2 and $\gamma$ secretase. B-AM, BAPTA-AM; Glc, glucose; rCalpain2, recombinant calpain 2 ; rIR, recombinant IR. $* * p<0.01, * * * p<0.001$
Fig. 2 Calpain 2 release with exosomes. Following incubation with glucose (Glc) for $96 \mathrm{~h}$, cells were incubated for an additional $24 \mathrm{~h}$ in (a) serum-free DMEM $(n=6)$ or (b) serum-free DMEM with or without $5 \mu \mathrm{mol} / 1 \mathrm{DON}$ $(n=3-7)$, prior to preparation of exosomes from media and cell lysates. White bars, $5.5 \mathrm{mmol} / 1$ glucose; black bars, $16.5 \mathrm{mmol} / 1$ glucose. $* p<0.05, * * * p<0.001$ a
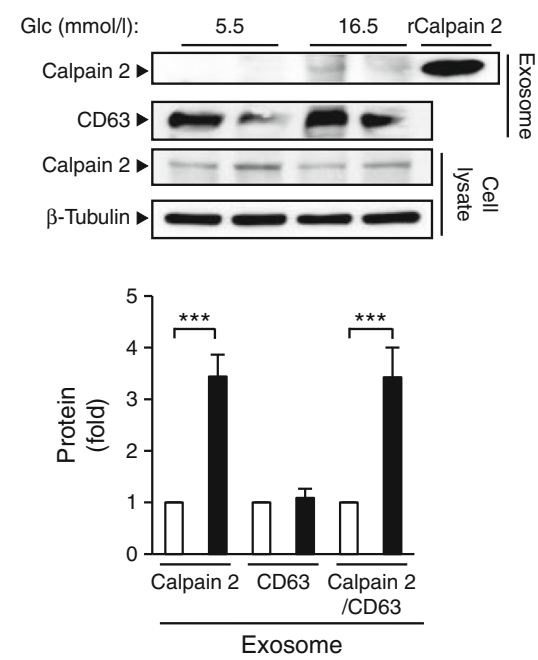

b
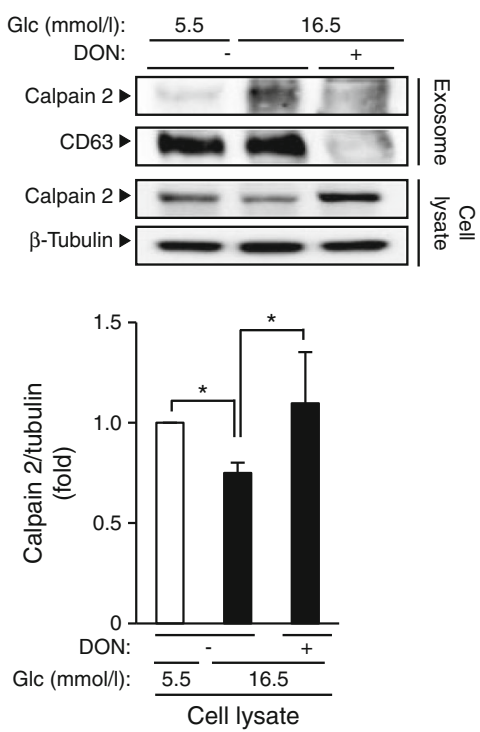
a

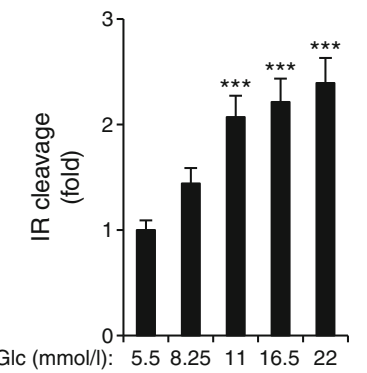

b

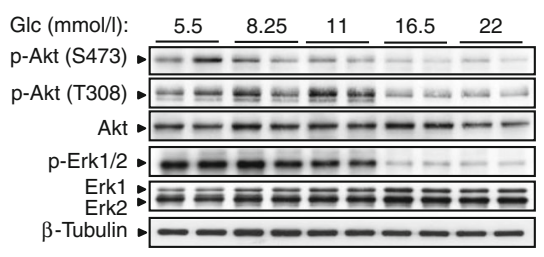

d
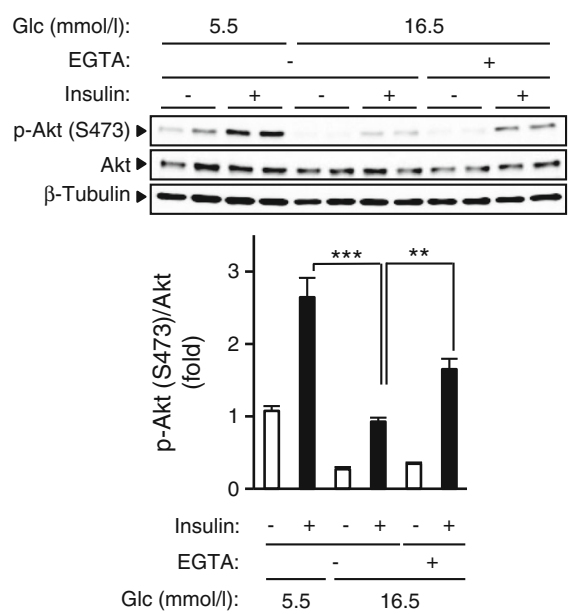

C

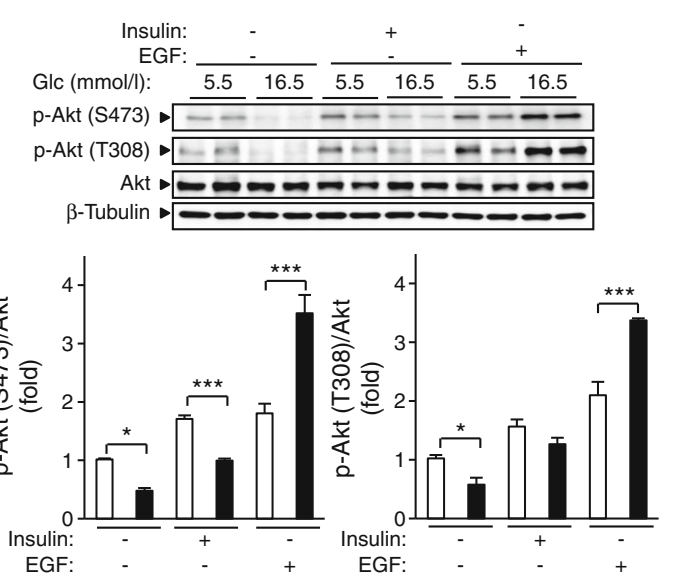

e
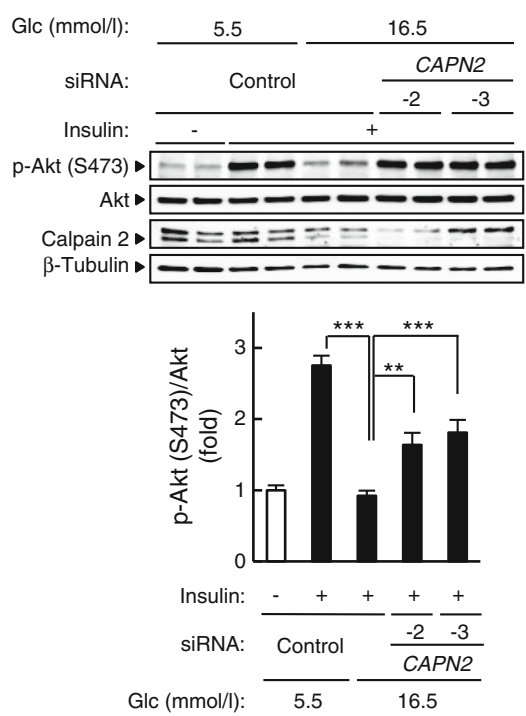

f
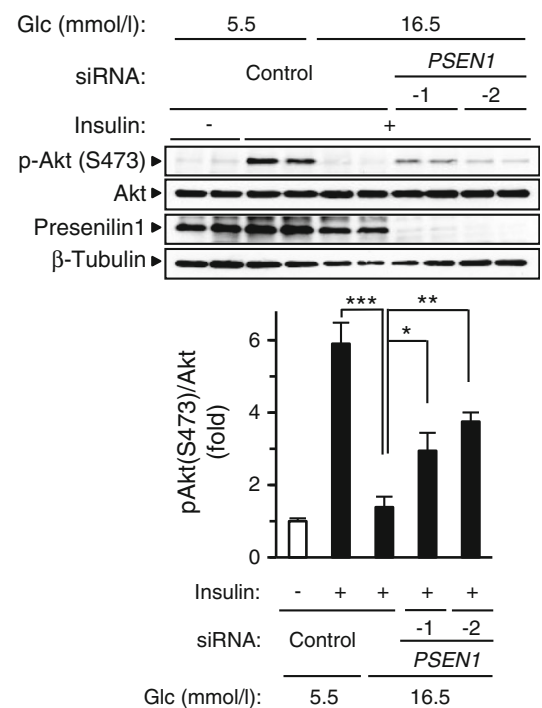

Fig. 3 IR cleavage impairs the insulin receptor signalling pathway. (a) IR cleavage relative to control $(5.5 \mathrm{mmol} / \mathrm{l}$ glucose). Following incubation with glucose for $72 \mathrm{~h}$, cells were incubated for an additional $24 \mathrm{~h}$, prior to media preparation $(n=11)$. (b) Impaired insulin receptor signalling. Following incubation with glucose for $72 \mathrm{~h}$, cells were incubated for an additional $24 \mathrm{~h}$, prior to cell lysate preparation. (c, d) Specific inhibition of insulin receptor signalling. Following incubation with glucose for $72 \mathrm{~h}$, cells were incubated in (c) serum-free DMEM, or (d) serum-free DMEM

\section{Metformin suppresses IR cleavage and ameliorates high-} glucose-induced impairment of insulin signalling Biguanides, including buformin and especially metformin, are known to improve insulin sensitivity and are widely used as the first-line drug for type 2 diabetes [23]. Based on the hypothesis that these drugs inhibit IR cleavage, we evaluated them in our model. Both drugs significantly suppressed the high-glucoseinduced increase in IR cleavage in HepG2 cells (Fig. 4a, b). Metformin suppressed the exosome-associated calpain 2 release induced by high-glucose treatment (Fig. 4c). Concomitantly, with or without $5 \mathrm{mmol} / \mathrm{l} \mathrm{EGTA}$ for $5 \mathrm{~h}$, and subsequently stimulated with (c, d) $10^{-9} \mathrm{~mol} / \mathrm{l}$ insulin or (c) $500 \mathrm{ng} / \mathrm{ml} \mathrm{EGF}$ for $30 \mathrm{~min}$. (c) $n=4-6$; (d) $n=4$. (e, f) Enhanced insulin sensitivity by inhibition of IR cleavage. Following knockdown with siRNA and incubation with glucose for $72 \mathrm{~h}$, cells were stimulated with $10^{-9} \mathrm{~mol} / 1$ insulin for $30 \mathrm{~min}(n=3-5)$. White bars, $5.5 \mathrm{mmol} / 1$ glucose; black bars, $16.5 \mathrm{mmol} / 1$ glucose. Glc, glucose. $* p<0.05, * * p<0.01, * * * p<0.001$

metformin restored phosphorylation levels of IRS1, Akt and ERK, which were suppressed by high glucose (Fig. 4d, g), and enhanced insulin-mediated Akt phosphorylation (Fig. 4e).

To determine whether the IR signal is responsible for the cellular insulin resistance induced by IR cleavage, the cell surface's IR content and tyrosine phosphorylation of IR $\beta$ following sIR generation were investigated (Fig. 4f). The cell surface expression and autophosphorylation of IR $\beta$ were not significantly decreased following cleavage, indicating that impaired insulin signalling induced by IR cleavage is not a result of IR signalling 


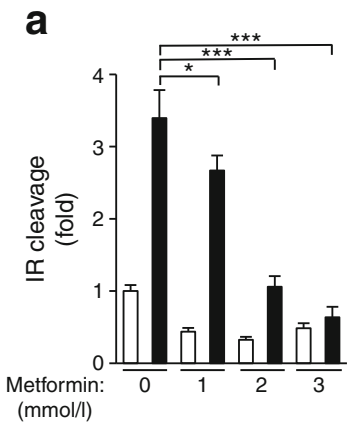

d

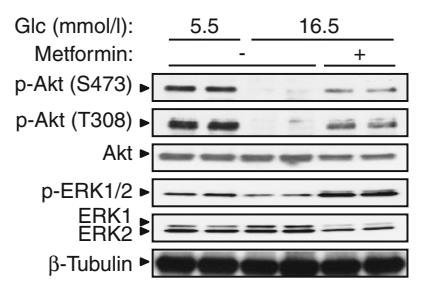

f

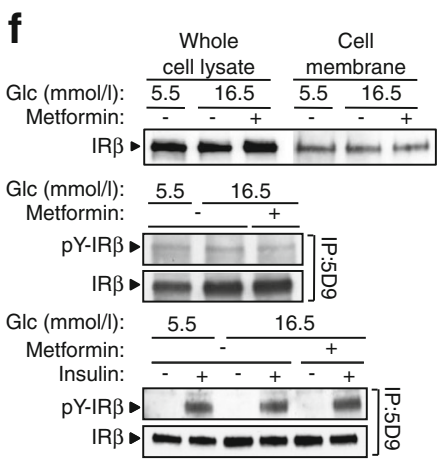

b

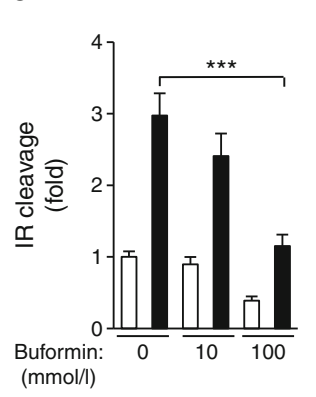

C

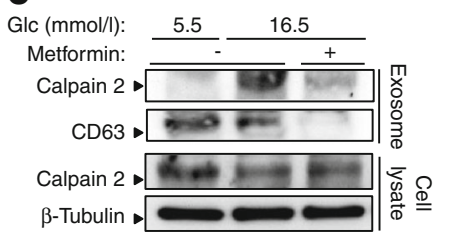

e

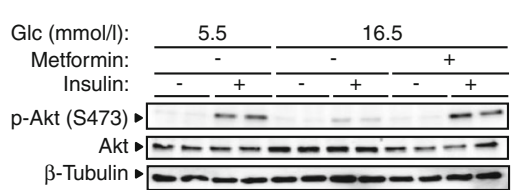

g h
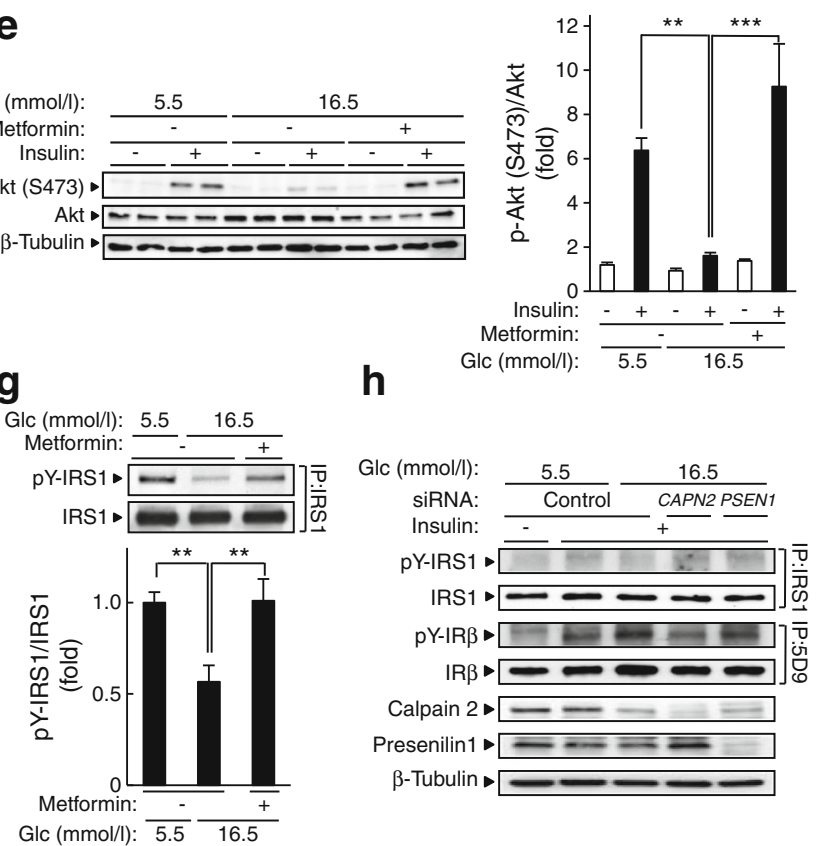

$\beta$-Tubulin

$5.5-16.5$

$$
\text { 薏 }
$$

i

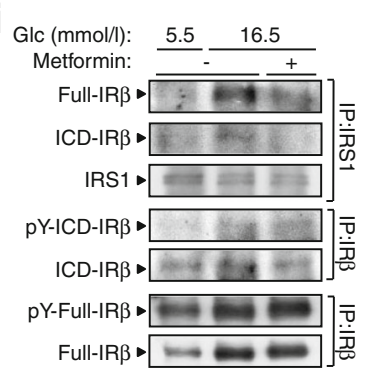

Fig. 4 Pharmacological inhibition of IR cleavage using metformin ameliorates high-glucose-induced impairment of insulin signalling. (a, b) IR cleavage relative to control (control conditions: $5.5 \mathrm{mmol} / 1$ glucose without (a) metformin or (b) buformin). Following incubation with glucose and the indicated concentrations of metformin or buformin for $72 \mathrm{~h}$, cells were incubated for an additional $24 \mathrm{~h}$ prior to media and cell lysate preparation $(n=8-9)$. (c) Inhibition of exosome release by metformin. Following incubation with glucose with or without $3 \mathrm{mmol} / \mathrm{l}$ metformin for $96 \mathrm{~h}$, cells were incubated in serum-free DMEM for an additional $24 \mathrm{~h}$ and then exosomes from media and cell lysates were prepared. (d, e) Enhanced insulin receptor signalling by metformin. (d, e) Cells $(n=4)$ were incubated with glucose with or without $3 \mathrm{mmol} / 1$ metformin for $96 \mathrm{~h}$, (e) followed by incubation in serum-free DMEM for $1 \mathrm{~h}$ and subsequently stimulation with $10^{-9} \mathrm{~mol} / \mathrm{l}$ insulin for $30 \mathrm{~min}$. (f) IR signalling on the cell surface. Cells were incubated with glucose with or without $3 \mathrm{mmol} / \mathrm{l}$ metformin for $96 \mathrm{~h}$, followed by biotinylation of cell surface j

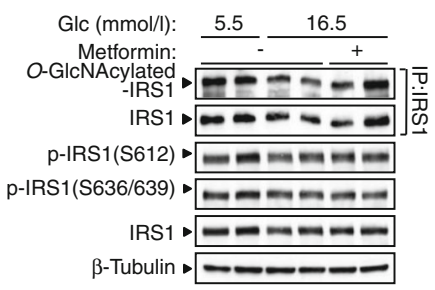

proteins, preparation of cell lysates, or stimulation with $10^{-8} \mathrm{~mol} / \mathrm{l}$ insulin for $30 \mathrm{~min}(n=2-3)$. (g) Enhanced IRS1 signalling by metformin. Cells were incubated with glucose with or without $3 \mathrm{mmol} / \mathrm{l} \mathrm{metformin} \mathrm{for} 96 \mathrm{~h}$ $(n=7-8)$. (h) Enhanced IRS1 signalling by inhibition of IR cleavage. Following knockdown with siRNA and incubation with glucose for $96 \mathrm{~h}$, cells were stimulated with insulin at $10^{-9} \mathrm{~mol} / \mathrm{l}$ (for pY-IRS1 analysis) or $10^{-8} \mathrm{~mol} / 1$ (for $\mathrm{pY}$-IR $\beta$ analysis) for $30 \mathrm{~min}$. $(n=3)$ (i) ICD-IR $\beta$ binding to IRS1. Following incubation with glucose with or without $3 \mathrm{mmol} / 1$ metformin for $96 \mathrm{~h}$, cells were incubated with $5 \mu \mathrm{mol} / 1$ MG132 for an additional $24 \mathrm{~h}(n=3)$. (j) $O$-GlcNAcylation and serine phosphorylation of IRS1. Cells were incubated with glucose with or without $3 \mathrm{mmol} / 1 \mathrm{metformin}$ for $96 \mathrm{~h}(n=3-4)$. White bars, $5.5 \mathrm{mmol} / 1$ glucose; black bars, $16.5 \mathrm{mmol} / 1$ glucose. Glc, glucose; IP, immunoprecipitation; pY, phosphorylated tyrosine. $* p<0.05, * * p<0.01$, $* * * p<0.001$ 
on the cell surface. Furthermore, inhibition of IR cleavage by knockdown of putative proteinases enhanced tyrosine phosphorylation of IRS1, but not of IR (Fig. 4h). Collectively, these results indicate that IR cleavage suppresses the insulin signalling pathway via decreased tyrosine phosphorylation of IRS1.

Next, we examined the interaction between the IR and IRS1. The ICD-IR $\beta$ was found to bind to IRS1, possibly exerting a dominant negative inhibitory effect on tyrosine phosphorylation of IRS1, despite the full length of the IR $\beta$ subunit binding to IRS1 in a glucose concentration-dependent manner (Fig. 4i). In addition, prolonged exposure to high glucose and metformin affected tyrosine phosphorylation of IRS1 without changing O-glycosylation and serine phosphorylation (Fig. 4j).

\section{Plasma sIR levels correlate with insulin resistance in pa-} tients with type 2 diabetes We have previously reported that type 2 diabetic patients exhibited a significantly elevated sIR level compared with controls [6]. The cellular insulin resistance observed in the in vitro model suggests physiological relevance of IR cleavage in vivo. Therefore, we investigated the association between plasma sIR levels and insulin sensitivity (determined using the euglycaemic-hyperinsulinaemic clamp technique) in patients with type 2 diabetes. We found that plasma sIR levels significantly correlated with the glucose clearance rate $\left(r^{2}=0.374, p=0.012\right.$; Fig. 5$)$.

\section{Discussion}

Here, we identified calpain 2 and $\gamma$-secretase as proteases that cleave IR and modulate cellular insulin signalling. It is generally accepted that calpain 2 exists and functions in the cytoplasm, but its activity requires a millimolar concentration of calcium to be fully active in vitro [24]. Calpain 2 does not contain a signal peptide which is necessary for the regulated secretory pathway, but nevertheless it has been reported to exist in the extracellular spaces of various tissues [25-27]. Exosomes, a type of extracellular vesicle, are derived from the endosomal pathway. The

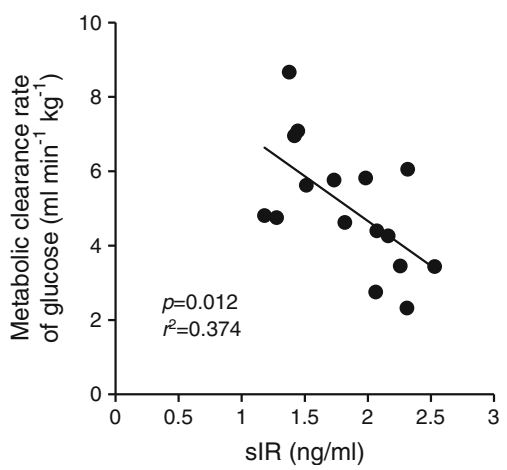

Fig. 5 Plasma sIR levels correlate with insulin resistance in patients with diabetes. The association between sIR levels and insulin sensitivity as assessed by the euglycaemic-hyperinsulinaemic clamp test in patients with type 2 diabetes $(n=16)$ multivesicular body proceeds to release intraluminal vesicles, such as exosomes, to the extracellular space instead of to the lysosomal degradation pathway [28]. Accumulating evidence shows that exosomes can modulate the cell environment and intracellular communications [29]. Calpain 2 has previously been identified in clathrin-coated vesicles [30], implying that it may be internalised in the endosomal pathway and consequently released into the extracellular space. This study identified for the first time that the extracellular existence of calpain 2 is associated with exosomes.

SIR contains a full IR $\alpha$ subunit, therefore its binding to insulin may reduce insulin sensitivity [31]. In the present study, in vitro, Akt signalling was suppressed in highglucose-treated HepG2 cells, despite washing the cells, changing the medium just prior to insulin treatment and using a ratio of insulin to sIR of approximately $10^{5}: 1$. In the clamp studies, the insulin concentration was about 100 times greater than that of sIR. Nevertheless, higher sIR levels correlated with lower insulin sensitivity. These results indicate that IR cleavage directly disrupts intracellular insulin signalling.

In this study, IR cleavage by calpain 2 and $\gamma$-secretase suppressed Akt phosphorylation and reduced tyrosine phosphorylation of IRS1. $O$-GlcNAcylation and serine phosphorylation of IRS1 have previously been reported to affect the downstream signalling of IRS1 [32, 33]; however, these modifications did not seem to be implicated in the impaired insulin signalling observed in our in vitro assay. Transient overexpression of the recombinant intracellular domain of IR $\beta$ did not induce a significant decrease in Akt phosphorylation (ESM Fig. 1), because of its lack of binding to IRS1 (ESM Fig. 2). Therefore, tyrosine phosphorylation of ICD-IR $\beta$, which has been postulated to be induced by cleavage of the extracellular domain of IR [34], is necessary for its binding to IRS1 and its inhibitory effect on insulin signalling.

IR cleavage potentially suppressed both basal and activated insulin signalling. This finding does not contradict the result that tyrosine phosphorylation of IRS1 was suppressed concomitantly with the increase in IR shedding since IRS1 protein deficiency has previously been reported to suppress basal and insulin-induced Akt activity in primary hepatocytes [35].

The identification of IR cleavage in HepG2 cells and primary hepatocytes indicates that the liver may be an important source of sIR [7]. IRS1 signalling in the liver has been shown to be associated with glucose homeostasis [36]. Systemic insulin resistance observed in Irs 1-knockout mice was restored by the expression of IRS1 specifically in the liver, indicating that hepatic IRS1 signalling is crucial for systemic insulin sensitivity in mice [35]. In humans, impaired tyrosine phosphorylation of IRS1 in the liver has been suggested to contribute to insulin resistance [37]. Taken together, the clamp study results, showing a significant association between SIR and insulin sensitivity, indicate the possibility that IR cleavage in the liver could potentially induce insulin resistance in patients with type 2 diabetes. 
Fig. 6 Schematic representation of the proposed model of IR cleavage in the pathogenesis of diabetes. Rip, regulated intramembrane proteolysis

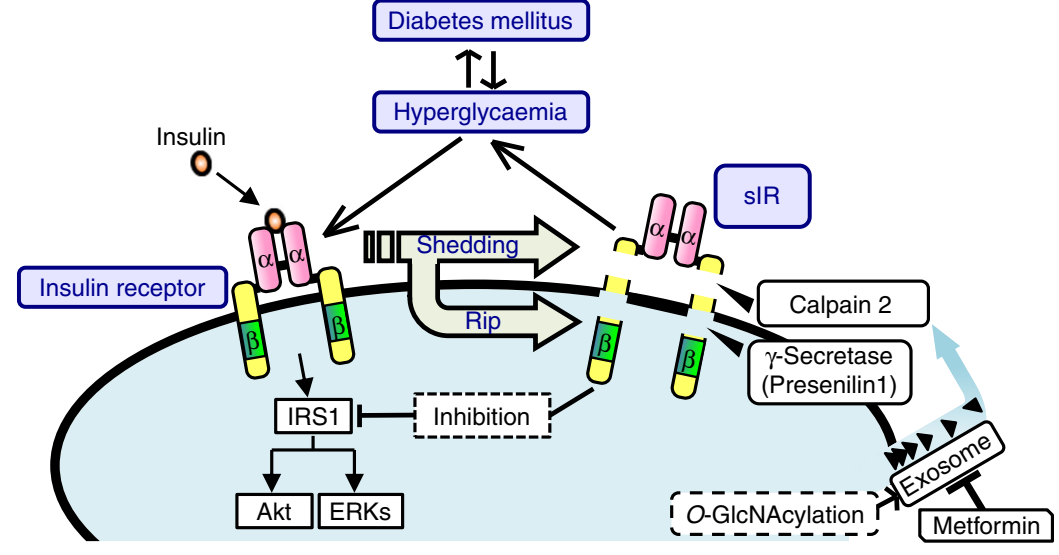

The molecular mechanism of metformin action is still controversial [38]. Metformin has been found to inhibit mitochondrial respiratory chain complex I, consequently increasing the AMP:ATP ratio, leading to activation of AMP-activated protein kinase (AMPK) and a reduction in gluconeogenic gene transcription [39]. In our model, the AMPK inhibitor compound C did not reduce the effects of metformin (ESM Fig. 3), indicating that it is unlikely that metformin suppresses IR cleavage via AMPK. Metformin has also been reported to decrease blood glucose levels independently of AMPK and liver kinase B1 [40]. Metformin has been shown to inhibit both adenylate cyclase, which induces suppression of glucagon signalling in hepatocytes [41], and mitochondrial glycerophosphate dehydrogenase, which suppresses endogenous glucose production [42]. It has also been shown to enhance insulin signalling through inhibition of the mechanistic target of rapamycin complex 1 (mTORC1) pathway [43]. The phosphorylation levels of mTOR and p70 S6 kinase (p70S6K) remained unchanged in response to high glucose or metformin treatment, indicating that the recovery of tyrosine phosphorylation of IRS by metformin is independent of mTORC1 signalling (ESM Fig. 4).

This study indicates that metformin prevents exosome release. Previous studies have reported that p53 induces tumour suppressor-activated pathway 6 (TSAP6) expression, which has been shown to increase exosome release [44], and that metformin decreases p53 expression in HepG2 cells [45]. In our model, no increments of p53 and TSAP6 were observed with high glucose, and metformin did not change the expression of these molecules (ESM Fig. 5), indicating that it is unlikely that metformin suppresses calpain 2 release through the p53-TSAP6 pathway. Although further study is necessary to identify the mechanism by which metformin regulates exosome-associated calpain 2 release, inhibition of IR cleavage offers a novel insulin-sensitising mechanism that is involved in the glucose-lowering effects of metformin.

Our findings, which imply that IR cleavage by a calciumdependent protease, calpain 2, modulates insulin signalling in vitro and insulin resistance in vivo, may be relevant to the molecular mechanism of chelation therapy using EDTA.
Chelation therapy in humans is known to improve insulin sensitivity and induce hypoglycaemia [46], effects that can be reversed by a calcium injection [47]. This observation could be explained by the results presented here and not by any conventional theories related to insulin sensitivity.

In summary, we have shown that exosome-associated calpain 2 cleaves IR extracellularly, and that sequential cleavage of IR by $\gamma$-secretase impairs the phosphorylation cascade of insulin signalling. This effect was ameliorated by metformin in HepG2 cells. The existence of SIR in human blood strongly suggests that IR cleavage modulates insulin signalling in vivo, whilst findings from the clamp study indicate that IR cleavage is associated with peripheral insulin resistance in patients with diabetes. These findings may establish a new framework in which IR cleavage regulates glucose homeostasis. The molecular mechanism underlying IR cleavage may help to explain the pathogenesis of insulin resistance and provide a new therapeutic target for type 2 diabetes (Fig. 6).

Acknowledgements We thank: Y. Ebina (Tokushima University), for his advice and discussion, T. Saitoh (Tokushima University), for help with conducting the study and K. Sakai (Tokushima University), for her technical assistance. We also thank Edanz Group Japan (Fukuoka, Japan) for help with revising the language of the manuscript.

Funding This work was supported by the Japan Society for the Promotion of Science KAKENHI, grant numbers 22790317, 24790317, 15K09391 (to TY), 25870483 (to KA-S) and 24790929 (to SI).

Duality of interest The authors declare that there is no duality of interest associated with this manuscript.

Contribution statement TY was responsible for conceptualising the study, data collection, analysis and interpretation, writing the manuscript and the integrity of the whole work. KA-S, SI and SH contributed to data collection, analysis and the writing of the manuscript concerning the in vitro study. AK and MM contributed to data collection, analysis, interpretation and the writing of the manuscript concerning the clinical study. MT, TM and HK contributed to data interpretation and the writing of the manuscript concerning the clinical study. All of the authors approved the final version of the manuscript. TY is the guarantor of this work. 


\section{References}

1. George S, Rochford JJ, Wolfrum C et al (2004) A family with severe insulin resistance and diabetes due to a mutation in AKT2. Science 304:1325-1328

2. Taira M, Taira M, Hashimoto N et al (1989) Human diabetes associated with a deletion of the tyrosine kinase domain of the insulin receptor. Science 245:63-66

3. Guo S (2014) Insulin signaling, resistance, and the metabolic syndrome: insights from mouse models into disease mechanisms. J Endocrinol 220:T1-T23

4. Bunner AE, Chandrasekera PC, Barnard ND (2014) Knockout mouse models of insulin signaling: relevance past and future. World J Diabetes 5:146-159

5. Taniguchi CM, Emanuelli B, Kahn CR (2006) Critical nodes in signalling pathways: insights into insulin action. Nat Rev Mol Cell Biol 7:85-96

6. The Soluble Insulin Receptor Study Group (2007) Soluble insulin receptor ectodomain is elevated in the plasma of patients with diabetes. Diabetes 56:2028-2035

7. Yuasa T, Amo K, Ishikura S et al (2014) Development of in vitro model of insulin receptor cleavage induced by high glucose in HepG2 cells. Biochem Biophys Res Commun 445:236-243

8. Orlando S, Polentarutti N, Mantovani A (2000) Selectivity release of the type II decoy IL-1 receptor. Cytokine 12:1001-1006

9. Raucci A, Cugusi S, Antonelli A et al (2008) A soluble form of the receptor for advanced glycation endproducts (RAGE) is produced by proteolytic cleavage of the membrane-bound form by the sheddase a disintegrin and metalloprotease 10 (ADAM10). FASEB J 22:3716-3727

10. Liu C, Xu P, Lamouille S, Xu J, Derynck R (2009) TACE-mediated ectodomain shedding of the type I TGF-beta receptor downregulates TGF-beta signaling. Mol Cell 35:26-36

11. Lin SY, Makino K, Xia W et al (2001) Nuclear localization of EGF receptor and its potential new role as a transcription factor. Nat Cell Biol 3:802-808

12. Brou C, Logeat F, Gupta $\mathrm{N}$ et al (2000) A novel proteolytic cleavage involved in Notch signaling: the role of the disintegrinmetalloprotease TACE. Mol Cell 5:207-216

13. Roth RA, Cassell DJ, Wong KY, Maddux BA, Goldfine ID (1982) Monoclonal antibodies to the human insulin receptor block insulin binding and inhibit insulin action. Proc Natl Acad Sci U S A 79: 7312-7316

14. Yuasa T, Uchiyama K, Ogura Y et al (2009) The Rab GTPaseactivating protein AS160 as a common regulator of insulin- and Galphaq-mediated intracellular GLUT4 vesicle distribution. Endocr J 56:345-359

15. Umehara A, Nishioka M, Obata T, Ebina Y, Shiota H, Hashida S (2009) A novel ultra-sensitive enzyme immunoassay for soluble human insulin receptor ectodomain and its measurement in urine from healthy subjects and patients with diabetes mellitus. Clin Biochem 42:1468-1475

16. Meyer TS, Lamberts BL (1965) Use of coomassie brilliant blue R250 for the electrophoresis of microgram quantities of parotid saliva proteins on acrylamide-gel strips. Biochim Biophys Acta 107:144-145

17. duVerle D, Takigawa I, Ono Y, Sorimachi H, Mamitsuka H (2010) CaMPDB: a resource for calpain and modulatory proteolysis. Genome Inform 22:202-213

18. Landman N, Kim TW (2004) Got RIP? Presenilin-dependent intramembrane proteolysis in growth factor receptor signaling. Cytokine Growth Factor Rev 15:337-351

19. Calzolari A, Raggi C, Deaglio S et al (2006) TfR2 localizes in lipid raft domains and is released in exosomes to activate signal transduction along the MAPK pathway. J Cell Sci 119:4486-4498
20. Nakajima K, Yamauchi K, Shigematsu S et al (2000) Selective attenuation of metabolic branch of insulin receptor downsignaling by high glucose in a hepatoma cell line, HepG2 cells. J Biol Chem 275:20880-20886

21. Gandy JC, Rountree AE, Bijur GN (2006) Akt1 is dynamically modified with O-GlcNAc following treatments with PUGNAc and insulin-like growth factor-1. FEBS Lett 580:3051-3058

22. Li YM, Xu M, Lai MT et al (2000) Photoactivated gamma-secretase inhibitors directed to the active site covalently label presenilin 1 . Nature 405:689-694

23. Inzucchi SE, Bergenstal RM, Buse JB et al (2012) Management of Hyperglycemia in Type 2 Diabetes: A Patient-Centered Approach: position Statement of the American Diabetes Association (ADA) and the European Association for the Study of Diabetes (EASD). Diabetes Care 35:1364-1379

24. Suzuki K, Hata S, Kawabata Y, Sorimachi H (2004) Structure, activation, and biology of calpain. Diabetes 53(Suppl 1):S12-S18

25. Schmaier AH, Bradford HN, Lundberg D, Farber A, Colman RW (1990) Membrane expression of platelet calpain. Blood 75:12731281

26. Nishihara H, Nakagawa Y, Ishikawa H, Ohba M, Shimizu K, Nakamura T (2001) Matrix vesicles and media vesicles as nonclassical pathways for the secretion of m-Calpain from MC3T3-E1 cells. Biochem Biophys Res Commun 285:845-853

27. Adachi E, Mukaiyama T, Sasai K et al (1990) Immunohistochemical evidence of the extracellular localization of calcium-activated neutral protease (CANP) in rabbit skeletal muscle, lung and aorta. Arch Histol Cytol 53:413-422

28. Urbanelli L, Magini A, Buratta S et al (2013) Signaling pathways in exosomes biogenesis, secretion and fate. Genes 4:152-170

29. Cocucci E, Meldolesi J (2015) Ectosomes and exosomes: shedding the confusion between extracellular vesicles. Trends Cell Biol 25: 364-372

30. Sato K, Saito Y, Kawashima S (1995) Identification and characterization of membrane-bound calpains in clathrin-coated vesicles from bovine brain. Eur J Biochem 230:25-31

31. Kanezaki Y, Matsushima R, Obata T, Nakaya Y, Matsumoto T, Ebina Y (2003) Injection of the insulin receptor alpha subunit increases blood glucose levels in mice. Biochem Biophys Res Commun 309:572-577

32. Yang X, Ongusaha PP, Miles PD et al (2008) Phosphoinositide signalling links O-GlcNAc transferase to insulin resistance. Nature 451:964-969

33. Boura-Halfon S, Zick Y (2009) Phosphorylation of IRS proteins, insulin action, and insulin resistance. Am J Physiol Endocrinol Metab 296:E581-E591

34. Shoelson SE, White MF, Kahn CR (1988) Tryptic activation of the insulin receptor. Proteolytic truncation of the alpha-subunit releases the beta-subunit from inhibitory control. J Biol Chem 263:48524860

35. Ueki K, Yamauchi T, Tamemoto H et al (2000) Restored insulinsensitivity in IRS-1-deficient mice treated by adenovirus-mediated gene therapy. J Clin Invest 105:1437-1445

36. Taniguchi CM, Ueki K, Kahn R (2005) Complementary roles of IRS- 1 and IRS-2 in the hepatic regulation of metabolism. J Clin Invest 115:718-727

37. Aytug S, Reich D, Sapiro LE, Bernstein D, Begum N (2003) Impaired IRS-1/PI3-kinase signaling in patients with HCV: a mechanism for increased prevalence of type 2 diabetes. Hepatology 38: 1384-1392

38. Rena G, Pearson ER, Sakamoto K (2013) Molecular mechanism of action of metformin: old or new insights? Diabetologia 56:18981906

39. Zhou G, Myers R, Li Y et al (2001) Role of AMP-activated protein kinase in mechanism of metformin action. J Clin Invest 108:11671174 
40. Foretz M, Hebrard S, Leclerc J et al (2010) Metformin inhibits hepatic gluconeogenesis in mice independently of the LKB1/AMPK pathway via a decrease in hepatic energy state. J Clin Invest 120:2355-2369

41. Miller RA, Chu Q, Xie J, Foretz M, Viollet B, Birnbaum MJ (2013) Biguanides suppress hepatic glucagon signalling by decreasing production of cyclic AMP. Nature 494:256-260

42. Madiraju AK, Erion DM, Rahimi Y et al (2014) Metformin suppresses gluconeogenesis by inhibiting mitochondrial glycerophosphate dehydrogenase. Nature 510:542-546

43. Kalender A, Selvaraj A, Kim SY et al (2010) Metformin, independent of AMPK, inhibits mTORC1 in a rag GTPase-dependent manner. Cell Metab 11:390-401
44. Yu X, Harris SL, Levine AJ (2006) The regulation of exosome secretion: a novel function of the p53 protein. Cancer Res 66: 4795-4801

45. Nelson LE, Valentine RJ, Cacicedo JM, Gauthier MS, Ido Y, Ruderman NB (2012) A novel inverse relationship between metformin-triggered AMPK-SIRT1 signaling and $\mathrm{p} 53$ protein abundance in high glucose-exposed HepG2 cells. Am J Physiol Cell Physiol 303:C4-C13

46. Meltzer LE, Palmon FP Jr, Kitchell JR (1961) Hypoglycaemia induced by disodium ethylenediamine tetraacetic acid. Lancet 2:637638

47. Walker M (1990) The chelation way : the complete book of chelation therapy. Avery, New York 